DOCUMENTOS 



\title{
Osmo Kivinen
}

Universidad de Turku, Finlandia

Osmo.kivinen@utu.fi

Tero Piiroinen

Universidad de Turku, Finlandia

\section{Hacia un relacionalismo metodológico pragmático. De la filosofización de la sociología a la sociologización de la filosofía ${ }^{1}$}

\author{
Toward pragmatist methodological relationalism. From \\ philosophizing sociology to sociologizing philosophy
}

Para um relacionismo metodológico pragmático. Da filosofização da sociologia à sociologização da filosofia

Artículo de investigación científica recibido el 02/03/2010 y aprobado el 31/05/2010

1 Nota del editor: este artículo de los profesores Osmo Kivinen y Tero Piiroinen de la Universidad de Turu, en Finlandia, apareció publicado por la revista CS en su número anterior (No. 5, enero -julio de 2010). En esa oportunidad el artículo se publicó con algunos problemas de edición y corrección de estilo, ajenos a la voluntad de los autores, que entorpecían su lectura y fácil comprensión, Por esta razón la revista $C S$ se disculpa con los autores y vuelve a publicar, con los ajustes pertinentes, este valioso trabajo que ellos amablemente nos cedieron para difundirlo en español. 



\title{
Resumen
}

En el presente artículo se analizan los enfoques relacionalistas de las ciencias sociales en términos de una distinción conceptual entre la "filosofización de la sociología" y la "sociologización de la filosofía". Estos marcan dos actitudes diferentes con respecto a la metafísica filosófica y sus compromisos ontológicos. El pragmatismo de los autores tiene su origen en el relacionalismo metodológico de Dewey, el cual se compara con los enfoques realistas con un compromiso ontológico, así como con el relacionalismo metodológico de Bourdieu. Se argumenta que la filosofía pragmática de las ciencias sociales es una herramienta adecuada para ayudar a los científicos sociales en su labor metodológica, en particular en lo que respecta a los estudios de casos basados en problemas.

Palabras clave: Metafísica, Pragmatismo, Realismo, Relacionalismo

\begin{abstract}
In this article, relationalist approaches to social sciences are analyzed in terms of a conceptual distinction between "philosophizing sociology" and "sociologizing philosophy". These mark two different attitudes toward philosophical metaphysics and ontological commitments. The authors' own pragmatist methodological relationalism of Deweyan origin is compared with ontologically committed realist approaches, as well as with Bourdieuan methodological relationalism. It is argued that pragmatist philosophy of social sciences is an appropriate tool for assisting social scientists in their methodological work, especially as regards problem-driven case studies.
\end{abstract}

Key words: Metaphysics, Pragmatism, Realism, Relationalism

\section{Resumo}

No presente artigo são analisadas as abordagens relacionistas das ciências sociais em termos de uma distinção conceitual entre a "filosofização da sociologia" e a "sociologização da filosofia”. Estes marcam duas atitudes diferentes no que diz respeito à metafísica filosófica e a seus compromissos ontológicos. O pragmatismo dos autores tem suas origens no relacionismo metodológico de Dewey, o qual é comparado com abordagens realistas a um compromisso ontológico, assim como com o relacionismo metodológico de Bourdieu. Aqui se argumenta que a filosofia pragmática das ciências sociais é uma ferramenta adequada para ajudar os cientistas sociais em seu trabalho metodológico, em particular no que diz respeito a estudos de casos baseados em problemas.

Palavras-chave: Metafísica, Pragmatismo, Realismo, Relacionismo 



\section{Introducción ${ }^{2}$}

En la actualidad, un número considerable de científicos sociales se identifican a sí mismos como relacionalistas, en un sentido u otro. Para citar algunos ejemplos, Mustafá Emirbayer (1997) escribió un manifiesto a favor de una sociología relacional; Margaret Somers (1998) reseñó un enfoque pragmático-realista a la relacionalidad en las ciencias sociales; y Stephan Fuchs (2001) planteó una teoría relacionalista de redes para la cultura y la sociedad. Por otra parte, el enfoque realista morfogenético de Margaret Archer (1995, 9ss., 165ss.) se fundamenta en una idea de propiedades emergentes relacionales y, por supuesto, el concepto central de campo social de Pierre Bourdieu quiere decir "una red, o configuración, de relaciones objetivas entre posiciones" (Bourdieu y Wacquant de 1992, 97). Por último, los autores de este artículo han denominado su propio punto de vista, de origen pragmático, como relacionalismo metodológico (Kivinen y Piiroinen 2004; Kivinen y Ristelä 2001). ${ }^{3}$

Entre los enfoques relacionalistas existen diferencias considerables que podrían ser analizadas de maneras muy diversas. En este artículo, se analizan en términos de dos actitudes opuestas que una investigación puede adoptar con relación a la metafísica -o sea, con respecto a "preguntas sobre la realidad que van más allá o detrás de lo que es capaz de ser abordado por los métodos científicos" (véase: Diccionario Oxford de Filosofía de 1996, 240; Diccionario Cambridge de Filosofía de 1999, 563).

Este par de actitudes opuestas es conceptualizado como la "filosofización de la sociología" y la "sociologización de la filosofía". En este caso, nuestra intención no es sólo jugar con las palabras sino, más bien, contrastar dos maneras diferentes de comprender la importancia de la ontología filosófica en la investigación científica social. Mientras que los que filosofizan la sociología asumen que el conocimiento de la realidad se debe basar en ciertos compromisos ontológicos de un tipo de metafísica tradicional, quienes sociologizan la filosofía establecen el hecho de que toda investigación es en el fondo una actividad social y concluyen que se puede hacer investigación sin ningún compromiso a priori con una ontología metafísica. La dicotomía existente entre pensadores sin y con compromiso ontológico

2 Nota del editor: CS agradece a los profesores Osmo Kivinen y Tero Piiroinen de la Universidad de Turu, en Finlandia, por conceder el permiso para la traducción y publicación de este artículo en nuestra revista. Este trabajo de los profesores Kivinen y Piiroinen apareció publicado originalmente en la revista Philosofy of the Social Sciences Volumen 36, Número 3, Septiembre 2006 1-27. Sage Publications. Traducción y revisión final de Enrique Rodriguez Caporali.

3 Este no es de ninguna manera un listado completo. Uno se encuentra con diferentes tipos de "relacionalismo" en diferentes ámbitos en las ciencias sociales, por ejemplo, en el campo de la política internacional (Jackson y Nexon, 1999), la economía (Bello, Chelariu y Zhang ,2003), y la antropología social historicista (Tilly, 2001). 
refleja la distinción entre realistas y pragmatistas en la filosofía. Mientras realistas como Margaret Archer $(1995,22)$, por ejemplo, están convencidos de que las investigaciones científicas sociales deben basarse en una ontología realista que "actúa tanto como portero y defensa de la metodología”, el relacionalismo pragmático detallado en el presente documento, que en su esencia está centrado en las acciones, es funcional y metodológico, renuncia por completo a la necesidad de un juego de lenguaje ontológico de tipo metafísico tradicional (véase Kivinen y Piiroinen, 2004, 2006).

Roy Bhaskar $(1979,1986)$ y John Searle $(1995,2001)$ son arquetipos de filósofos realistas que sugieren firmes compromisos ontológicos para los científicos sociales. Bhaskar (1986:6) propone un realismo metafísico, "[que]... depende de la factibilidad de una filosofía, a diferencia de la sociología o la historia de la ciencia; y, dentro de la filosofía, de una ontología, así como de una epistemología". Es en este sentido que Bhaskar (1979 : 17), fiel a su "perspectiva ontológica", la cual sienta unas bases ontológicas antes de cualquier trabajo metodológico, sugiere que el fundamento de las ciencias sociales se debe buscar respondiendo a la pregunta: "¿qué propiedades tienen las sociedades y las personas que podrían convertirlas en objetos de conocimiento para nosotros?” Y Searle (2001: 15) quiere establecer una rama de la filosofía que denomina "filosofía de la sociedad" -una minuciosa investigación ontológica de la naturaleza de la realidad social, destinada a captar "cómo la realidad social encaja en nuestra ontología global, es decir, cómo la existencia de hechos sociales se relaciona con otras cosas existentes" (Searle, 1995 : 5-6). Por el contrario, nuestro relacionalismo metodológico extrae sus experiencias de pensadores pragmáticos, especialmente de John Dewey (1982 [1920], [1922] 1983, 1984 [1929]), cuyos intereses filosóficos giraban, en una palabra, en torno a la comunidad, la fuente angular del idioma, las costumbres y los hábitos (Dewey [1922] 1983, 15-16, 31, 84). De acuerdo con Dewey (1982 [1920], 94), todo en la cultura humana (o toda "experiencia"), incluida la filosofía, debe ser contextualizado.

Además de la comparación entre pragmatismo y realismo, contrastamos nuestro relacionalismo metodológico inspirado por Dewey con el enfoque similar de Pierre Bourdieu (Bourdieu y Wacquant 1992, 15), argumentando que a pesar de que las teorías de Bourdieu sean sobre la práctica (Bourdieu 1977), siguen siendo poco prácticas para los trabajos de investigación real.

\section{Ontología relacional}

"En la actualidad, los sociólogos enfrentan un dilema fundamental: si se debe concebir el mundo social como algo conformado principalmente por sustancias 
o procesos, por "cosas" estáticas o por relaciones dinámicas y en desarrollo". Así comienza Mustafá Emirbayer (1997 : 281) su "Manifiesto a favor de una sociología relacional". Emirbayer, al igual que muchos otros relacionalistas (Bourdieu y Wacquant de 1992 : 228-30; Fuchs 2001 : 1; Somers, 1998 : 743 44), yuxtapone así el relacionalismo con el tipo de sustancialismo que busca las naturalezas intrínsecas de las cosas como "realmente son", independientemente de nuestras acciones y descripciones. En la crítica al substancialismo, Emirbayer se basa en las ideas de John Dewey, entre otros, para señalar que es totalmente inverosímil suponer que las investigaciones sociales van a revelar algunas entidades fijas o esencias verdaderas. En cambio, insta a renunciar a la idea de "unidades discretas pre-concebidas, tales como la persona o la sociedad, como puntos de partida para un análisis sociológico final" (Emirbayer, 1997 : 287; Elias, 1976, 1982; Tilly 1984, 2001).

Si existe una lección metodológica por aprender de la biología evolutiva es que sería infructífero aferrarse a la doctrina filosófica que afirma que hay alguna esencia característica distintiva en la naturaleza (Dennett 2003 : 127; Mayr 1982 :38-39). De esta manera, estamos de acuerdo que desde una perspectiva evolutiva no existe ninguna alternativa al relacionalismo. Sin embargo, a diferencia de Emirbayer (1997 : 282ff.) que se enfoca en "la ontología, en gran medida [...] agrupando cuestiones relacionadas con la epistemología," nos parece infructuoso pensar en el relacionalismo como algún tipo de doctrina ontológica. A menudo, los científicos sociales dan por sentada la idea que cualquier serie de descripciones implica algún tipo de ontología -y se comprometen con las descripciones que se infieren a partir de las que consideran las mejores teorías (Sheehy 2003: 198-99). Incluso pensadores con una conciencia histórica, como Charles Tilly, anhelan ocasionalmente modelos científicos sociales que "se basen en ontologías plausibles", es decir, en recuentos plausibles de "la naturaleza de lo que se va a conocer" (Tilly, 1995 : 1594-1602). Desde un punto de vista pragmático, es difícil comprender por qué un científico social debe construir sus investigaciones sobre estos supuestos metafísicos que por definición están "más allá [...] de ser abordados por los métodos científicos" (Diccionario Oxford de Filosofía 1996 : 240). Para ser verdaderamente autorreflexivos, las funciones inquisitivas de la investigación científica no se pueden desarrollar razonablemente a partir de misterios metafísicos que no se pueden resolver.

Mientras que la construcción de teorías con un alcance general, sugerida por Emirbayer (1997 :311), requiere una ontología global a priori-relacional- como base de las prácticas de investigación, otra relacionalista, Margaret Somers (1998), critica estos enfoques "orientados en la teoría” y sugiere más un punto de vista 
"orientado en el problema", a partir del cual la investigación científica social se entiende como estudios de casos encaminados a resolver problemas de investigación específicos y bien definidos que surgen a partir de problemas concretos que las personas enfrentan en su vida cotidiana. ${ }^{4}$ Todo nuestro conocimiento y razonamiento está siempre marcado por el impacto de nuestro época -es sólo en respuesta a cuestiones particulares en determinados momentos históricos que se interpretan los datos como evidencia de uno o otro punto de vista (Somers, $1998: 730-39)$.

Según Somers (1998: 758), los investigadores siempre trabajan dentro de los límites de los problemas que se han propuesto explicar; el modo pragmático de expresar nuestra propia idea sostiene que todo lo que se puede tener es el punto de vista de un actor-que nadie puede alejarse de sus propias acciones (Kivinen y Piiroinen 2004 : 233-34, 239, 241-42). Desafortunadamente, Somers adhiere a lo que denomina "realismo minimalista", una posición que depende de la ontología relacional realista. Para Somers (1998 : 766-67), esto significa sencillamente ser fiel a la idea de que existe un mundo social relacional independientemente de las creencias que se pudieran tener al respecto. En este sentido, las ideas de Somers son similares a las de otros pensadores que se pueden identificar como realistas pragmáticos, tales como Susan Haack (1993, 1998), Rom Harré (1986, 1997), Hilary Putnam $(1987,1990)$, Nicholas Rescher $(1996,2000)$ y Sandra Rosenthal $(2000,2003)$. Todos estos autores se encuentran en una línea pragmática convencidos de que la naturaleza del conocimiento está cargada de historia y está ligada a conceptos; sin embargo, como realistas, se comprometen con el juego del lenguaje metafísico de la ontología y con lo que podría denominarse una concepción "referencialista" del conocimiento (Kivinen y Piiroinen 2004 : 236).

Las actitudes ambivalentes de los realistas pragmáticos hacia la metafísica son captadas por Sandra Rosenthal (2003 : 50) quien afirma que al regalar los estáticos muebles ontológicos, uno debe rehusarse a "botar al bebe metafísico junto con la objetable agua de baño". La solución de Rosenthal es una versión de la "metafísica pragmática de procesos" que, en ciertos aspectos, es similar a la desarrollada en más detalle por Nicholas Rescher (1996) quien, por cierto, también es muy explícito al afirmar que la metafísica de procesos no es sino una variación del concepto de los "metafísicos tradicionales" de la "realidad como tal" -es decir, "el reino de lo que realmente es como verdaderamente es"- lo cual se debe contrastar con la mera "imagen de la realidad" que los seres humanos

4 El objetivo principal de la crítica de Somers es la metodología orientada en la teoría de los teóricos de la elección racional, como Edgar Kiser y Michael Hechter (1991), quienes acogen lo que Somers denomina una "empresa militantemente anti-positivista" de planteamientos tanto teóricos como ontológicos a priori (Somers, $1998: 723-26)$. 
podemos tener (Rescher 2000 : 133). Susan Haack (1998 : 149-66) opta por una selección más prudente de palabras, tratando de evitar los excesos del crudo realismo metafísico pero, también, rechazando, por ejemplo, el relativismo conceptual de Hilary Putnam como una tautología poco interesante o una absoluta contradicción. Y Putnam, por supuesto, es incluso más prudente que Haack al evitar el realismo metafísico, pero aún así parece aferrarse a la idea de algún tipo de ontología "pluralista" (Putnam, 1990 : 95). En cuanto a Somers y su relacionalismo orientado en los problemas, en el tema de la ontología, su posición es bastante cercana al realismo pragmático convencional de, por ejemplo, Rom Harré. Tanto Harré (1998 : 41) como Somers (1998 : 750) asumen que cualquier teoría del conocimiento presupone una elección más o menos explícita de alguna ontología.

No encontramos fructífero seguir a los pragmáticos realistas en sus intentos por reemplazar el mobiliario ontológico a priori orientado en la teoría por alguna ontología de procesos, ontología pragmática u ontología relacional con una denominación híbrida, pues los enfoques en este sentido continúan manteniendo muchas de las peculiaridades de los realismos relacionales orientados en la teoría, los cuales consideran que los objetos de investigación son las relaciones ontológicas independientes de las descripciones o las esencias relacionales de los objetos existentes. ${ }^{5}$ Margaret Archer (1995) es un buen ejemplo de una realista con un fuerte compromiso ontológico, dispuesta a ahondar en descripciones minuciosas sobre las diversas relaciones ontológicamente verdaderas que ve en el mundo social. De hecho, ella sostiene que cualquier teoría social viable debe basarse en una ontología social realista - de lo contrario "caer en el instrumentalismo es fatal" (Archer, 1995 : 161). Como realista ontológica (relacionalista), Archer (1995 : 136) se trepa "sobre los hombros teóricos" de Roy Bhaskar, filósofo que afirma que existen relaciones reales, no analíticas, interiores entre las cosas en el mundo en las que " $A$ no sería lo que es esencialmente a menos que $B$ estuviera relacionado con $A$ de la manera en que lo está" (Bhaskar, 1979: 54). ${ }^{6}$ Lo que encaja para Archer -o sea, que las ontologías relacionales se basan en un postulado a priori totalmente filosófico- debe ser vergonzoso para Somers, quien se opone convincentemente a una teorización ontológica a priori. De hecho, existe un cierto aire de incomodidad cuando Somers trata de separarse, a sí misma, del realismo crítico de Bhaskar, siguiéndolo al mismo tiempo al distinguir los

5 De hecho, incluso algunos de los realistas relacionales con una orientación ante todo pragmática se aferran a este tipo de pensamiento, afirmando que algunas cosas son definidas no por las personas, "sino por la naturaleza, por las relaciones en que se encuentran independientemente de nosotros”(Ryder, 2003 : 72).

6 En otros documentos hemos abordado la ontología realista crítica de Bhaskar (Kivinen y Piiroinen, 2004) y las diferentes distinciones ontologizadas de Archer (Kivinen y Piiroinen, 2006) más detalladamente. 
objetos teóricos, transitivos - producidos dentro de la ciencia- de los objetos reales e intransitivos de la ciencia. En cuanto a la relación entre estos objetos, Somers incluso reconoce que cree en el dogma representacionalista crucial -"en la importancia de establecer qué teorías representan más cercanamente la realidad" (Somers, 1998 : 743-45 y n. 16; Bhaskar, 1979 : 73-74; 1986 : 51-52).

En contraste, argumentamos a favor de un enfoque pragmático, anti-representacionalista y anti-referencialista que no se suscriba al dualismo sujeto-objeto cartesiano y que, por consiguiente, no necesita plantear conexiones referenciales ni representacionales entre sujeto y objeto. En su lugar, el punto de partida para nosotros los pragmáticos es la acción humana. Los conceptos pueden entenderse sencillamente como herramientas utilizadas por personas que actúan en sus intentos por lidiar con los problemas que enfrentan (Kivinen y Ristelä, 2002, 2003; Kivinen y Piiroinen 2004, 2006). No tiene sentido especular, como lo hace Bhaskar, entre una descripción independiente de las relaciones y una descripción independiente de lo que los objetos son esencialmente. Se debería abandonar la idea de que la ciencia revela la verdadera esencia de los objetos existentes. Como lo enseñó el pragmático John Dewey, hablar de esencias y accidentes como cosas inherentemente diferentes entre sí es simplemente repetir una antigua distinción ontológica que ha perdido su significado; decir que algo es "esencial" debe entenderse sencillamente como una afirmación sobre su "naturaleza indispensable en una investigación dada" (Dewey [1938] 1991, 141). Louis Menand ha captado bastante bien el enfoque de Dewey en términos de una analogía en la que, para Dewey, "las ideas y las creencias son lo mismo que las manos: instrumentos para hacerle frente a algo". Este es el motivo por el cual no tiene sentido suscitar dudas filosóficas sobre si el mundo puede ser conocido "correctamente", en el sentido de cruzar la división entre la mente y el mundo para representar las cosas de la realidad como realmente son. "Tiene tan poco sentido hablar de una "división" que se debe superar entre la mente y el mundo, como hablar de una "división' entre la mano y el medio ambiente" (Menand, 2001 : 361; Rorty, 1999 : xxiii). La superioridad de algunas descripciones en comparación con otras se debe a que son más adecuadas -mejores herramientas para resolver algunos problemas específicos (Rorty, 1999 : 47-71; Wittgenstein, 1953 : $\$ \$ 21-23,43$ ) y esta concepción de las descripciones es compatible con la percepción de que no hay forma de decidir cuáles de las muchas descripciones de un objeto captan sus "características intrínsecas" (Rorty, 1998 : 86).

Así, los objetos de investigación científica pueden entenderse como descripciones intencionales y connotativas en un campo de investigación científica, en términos de un juego de lenguaje científico. Los juegos de lenguaje, a su vez, están 
íntimamente relacionados con las prácticas. El lenguaje es una herramienta de comunicación y coordinación de acciones mediante el cual la gente puede hacer más predecibles el comportamiento y los resultados de las acciones propias y de los demás; el uso del lenguaje tiene lugar dentro de un contexto de prácticas compartidas. Todos los objetos de investigación son concebibles sólo cuando se describen lingüísticamente en términos de un juego de lenguaje, desde el punto de vista del actor, con respecto al ámbito del problema del investigador. Estamos de acuerdo con los realistas pragmáticos en que el valor de los conceptos y las teorías científicas (cargadas de historia) debe ser ponderado por su capacidad práctica para ayudar a solucionar problemas, pero a diferencia de ellos, no ligamos esta capacidad de los conceptos y las teorías, para la solución de problemas, con la idea de que son representaciones correctas de una realidad independiente de las descripciones. Al igual que Dewey ([1925] 1981, 173-225), abandonamos todo el dualismo sujeto-objeto, el cual primero presupone al sujeto cognoscente como una entidad distinta de los objetos de su conocimiento y, luego, se dedica a tratar de comprender cómo el sujeto podría formar representaciones correctas del mundo. Otros dualismos inquietantes que acompañan la distinción sujetoobjeto incluyen la dicotomía entre la materia prima de "contenido" empírico y el "esquema" conceptual que, supuestamente, divide la materia en rebanadas -lo cual es básicamente otra forma de expresarlo-, y el dualismo del mundo real de los hechos independientes del lenguaje y la mente que lo representa (Davidson, especialmente 1984, 189; 1990, 304). Abandonando estos dualismos, nos deshacemos de las disciplinas filosóficas conocidas como ontología y epistemología, pues la ontología es sólo una versión del objeto, del contenido, del mundo real independiente del lenguaje, y la epistemología es sólo una versión de la relación entre sujeto y objeto, esquema y contenido, mente y mundo (Rorty, 1997 : 149).

Perfectamente se puede argumentar a favor de la relacionalidad de los objetos de investigación a partir de una base que no sea ontológica. "En la ciencia, ya que los significados son determinados con base en su relación como significados para otro, las relaciones se convierten en objetos de investigación", señala Dewey (1991 [1938] : 119). Según el relacionalismo de redes de Stephan Fuchs (2001 : 2-3, 12-23), todo debería verse como relativo a los "observadores", los cuales a su vez son redes de distinciones relacionadas; tanto los observadores como las culturas son redes de comunicación y significado. A diferencia de aquellos que buscan fundamentos filosóficos para prácticas científicas, Fuchs (2001: 5) hace hincapié en que si seguimos a Wittgenstein y a los pragmáticos, no quedarán problemas que sean específicamente "filosóficos", sólo los problemas que surgen en momentos particulares, en culturas particulares. La filosofía no es más que 
una de esas culturas. De manera similar, la sociología de las ideas de Randall Collins (1998 : xviii, 1-2) parte de la comprensión relacional de que cualquier concepción intelectual puede explicarse como resultado de la dinámica de una red intelectual y que "el proceso comunicativo crea a los pensadores como nodos del proceso".

Tan pronto entendamos que ni la verdad ni la objetividad pueden escapar de los límites de las costumbres que utilizan el lenguaje de nuestra comunidad -que estos conceptos adquieren un significado inteligible sólo después de ser comprendidos dentro del contexto de la discusión y el debate de la comunidad sobre los problemas y del logro de acuerdos intersubjetivos sobre algunos de ellos- veremos que todas las ontologías realistas, tanto relacionales como sustancialistas, no deben ser más que los resultados de diálogos entre filósofos en el ámbito de la metafísica, en términos de un juego de lenguaje filosófico. En lugar de revelar la estructura de la realidad o las condiciones necesarias de las investigaciones científicas, solamente revelan las posiciones metafísicas a priori de quienes las sostienen. Pretender que esas posiciones ofrecen, de alguna manera, las bases necesarias para la investigación lleva, a menudo, a círculos viciosos, a batallas interminables de intuiciones a expensas de debates metodológicamente fructíferos que podrían dar como resultado el mejoramiento de nuestras prácticas científicas sociales (Van Bouwel 2004 : 304-5; Fuchs, 2001 : 6).

\section{Sociologización de la Filosofía}

Por definición, la filosofización de la sociología está ligada a la búsqueda de puntos de partida filosóficos a priori y de los fundamentos metafísicos de la sociología. Esto se debe contrastar con la sociologización de la filosofía-vincular cualquier actividad humana, incluida la filosofía, al contexto de una comunidad, una época y un lugar. Con esto no sugerimos que se deba intentar "resolver" los problemas filosóficos mediante los métodos de la sociología. De hecho, si algo ha establecido la filosofía en sus aproximadamente 2.500 años de existencia es el hecho de que cualesquiera que sean los problemas centrales en la filosofía, éstos

7 Sin embargo, para mayor claridad, se debe anotar que tanto Collins como Fuchs difieren del pragmatismo anti-representacionalista específico que estamos promoviendo aquí. Collins (1998: 858-62) defiende un tipo de "realismo constructivista", argumentando que el constructivismo es realmente una forma de realismo. Fuchs (2001 : 18-23), por su parte, enfatiza demasiado en la idea de un "observador", en vez de afirmar, como lo hacemos nosotros, que las descripciones son lo que son con relación a otras descripciones, Fuchs utiliza la antigua terminología empiricista y afirma que las observaciones son lo que son con relación a otras observaciones. Para Fuchs, son las observaciones lo que importa, y cualquier red de distinciones relacionadas es un posible observador, incluyendo, por ejemplo, especies de animales, bacterias, amantes y la disciplina de la física. Además, Collins $(1998,7,14-15,20)$ al igual que Fuchs $(2001,37,63-66)$ habla sobre "estructuras sociales" de una manera demasiado concreta que, consideramos, los pragmáticos prefieren evitar a fin de concentrarse en las actividades humanas reales. 
no pueden resolverse: ser irresoluble es un prerrequisito para que un problema sea verdaderamente "filosófico". Sin embargo, estos eternos problemas pueden elucidarse notablemente mediante explicaciones que los colocan en un contexto social adecuado y prestan atención al hecho de que los filósofos profesionales necesitan enfocarse en un sinnúmero de problemas que sólo pueden ser planteados y tratados en términos de los juegos de lenguaje a los que tienen acceso, si quieren asegurar la continuidad de su profesión como disciplina académica.

Nuestra tesis es que cuando se dedican al underlabouring metafísico los realistas, con un compromiso ontológico, están filosofizando la sociología; mientras que lo más probable es que los pragmáticos, con una orientación metodológica, estén sociologizando la filosofía. Los extremos de la filosofización de la sociología y la sociologización de la filosofía pueden ilustrarse mediante el debate entre el realista-racionalista John Searle y el neo-pragmatista Richard Rorty. Searle (1995 : 155) defiende el realismo como "una teoría ontológica" que, a pesar de no decirnos cómo son las cosas, nos dice que hay una manera en que son -independientemente del modo en que las describimos. Además, Searle (1993 : 57, 60-61) cree que el tipo de realismo que defiende está en la esencia de lo que él denomina "tradición racionalista occidental ", la cual, entre otras cosas, subyace a "la concepción occidental de la ciencia". Rorty (1998 : 64), a su vez, duda que las prácticas sociales tengan en realidad un fundamento filosófico. Se niega a tener una teoría ontológica, pues considera que no tiene caso tratar de responder preguntas filosóficas como: “¿es X verdaderamente real?”. Más allá de preguntas concretas y reconocibles como: “¿tiene X poder de predicción?”. Mientras que el primer tipo de preguntas es siempre objeto de debates interminables dentro de las comunidades de filósofos, el segundo tipo de preguntas es efectivamente decidible dentro de la comunidad científica pertinente (Rorty, 1997 : 149-51). Así que cuando Searle insiste en que "el sentido de la epistemología es facilitar una ontología que exista de forma independiente" y que, por consiguiente, se debe tener cuidado para no "confundir la epistemología con la ontología", Rorty responde que "los pragmatistas no tienen una epistemología ni una ontología, por eso no podemos confundirlas" (Rorty y Searle, 1999 : 45, 50).

Una ilustración idónea de la forma de pensar que llamamos filosofización de la sociología es el artículo de Searle titulado "Ontología social y filosofía de la sociedad" (2001), en el que exige una nueva rama de la filosofía, la filosofía de la sociedad. Searle se queja de que a menudo la filosofía de las ciencias sociales gira en torno sólo a preguntas sobre la metodología y ofrece su filosofía social como algo que "se centra esencialmente en preguntas de la ontología social". Según Searle, la filosofía de las ciencias sociales sería una instancia especial de esta 
nueva filosofía de la sociedad o realidad social con una orientación ontológica (Searle, 2001 : 15-16). Esta es una forma de pensar contraria a la que estamos sugiriendo -de concentrarse más exclusivamente en cuestiones metodológicas, dejando a un lado por completo las conjeturas metafísicas y reemplazándolas con preguntas de investigación operacionalizables (Fuchs, 2001 : 6). Lo que necesitan los científicos sociales que están realizando una investigación no es ontología filosófica sino, sencillamente, un acuerdo dentro de la comunidad pertinente acerca de cuál es el caso y qué hacer al respecto.

La interpretación siempre tiene lugar en una comunidad. Existe un argumento contextualista wittgensteiniano sobre la inteligibilidad: lo que hacemos y lo que decimos adquiere importancia sólo en un contexto proporcionado por una práctica, una forma de hacer las cosas que compartimos (Medina, 2003 : 300). Esta comprensión es lo que la posición de Wittgenstein tiene en común con la de Bourdieu (Medina : 2003), por una parte, y con de Dewey, por la otra (Medina : 2004). Como afirma Dewey:

Todo grupo cultural posee una serie de significados que están tan profundamente arraigados en sus costumbres, ocupaciones, tradiciones y formas de interpretar su entorno físico y la vida en grupo, que forman las categorías básicas del sistema del lenguaje mediante el cual se interpretan los detalles (1991 [1938] 68).

Las comunidades científicas no son la excepción a este aspecto; todo campo de la ciencia es un espacio de oportunidades que guía las indagaciones y separa a los profesionales de los aficionados. De la misma manera en que no existiría ninguna obra de arte independientemente de un campo del arte, una comunidad artística legimitizada (Bourdieu : 1993), tampoco habría hallazgos científicos sin una comunidad de usuarios del lenguaje reconocidos como legítimos científicamente. De hecho, en un campo científico tenemos las familiares prácticas y normas sociales que Joseph Rouse (1987 : 120) nos recuerda: los criterios de admisibilidad racional que están incorporados en las prácticas sociales institucionales en las que la investigación es arbitrada, revisada, citada, y canonizada en obras de referencia.

Mucho antes de Bourdieu y Rouse, e incluso antes de Wittgenstein, Dewey (1982 [1920] : 26-27) expresó la idea de sociologizar la filosofía, la naturaleza socialmente condicionada de toda investigación, incluida la filosofía. Hizo esto simplemente afirmando que toda investigación se desarrolla a partir de un trasfondo cultural. Para Dewey, las doctrinas filosóficas no indican realidades trascendentes, pues "la filosofía no se originó a partir de material intelectual, 
sino a partir de material social y emocional", y, por lo tanto, para entenderla, uno debe "ligar la historia de la filosofía con un estudio de la antropología, de la vida primitiva, de la historia de la religión, la literatura y las instituciones sociales" (Dewey [1920] 1982 : 93).

La perspectiva de Dewey sobre la ciencia y su supuesta "fundamentación" filosófica, entonces, consistió en destacar su naturaleza como actividad humana, como prácticas que son colectivas por naturaleza y que siempre dependen de ciertos antecedentes históricos. Aunque existen muchos, por ejemplo, entre los realistas pragmáticos relacionales que quisieran concebir a Dewey como un filósofo por excelencia, en el sentido de los metafísicos tradicionales, explicando las tendencias anti-representacionalistas de Dewey como señales solamente de la promoción de una especie de metafísica de procesos o un intento por sintetizar lo objetivo con lo subjetivo (Emirbayer, 1997; Margolis, 2003; Rescher, 1996; Rosenthal, 2003), nos parece que su verdadera innovación consistió en despejar el camino hacia la sociologización de la filosofía en vez de la filosofización de la sociología. Joseph Margolis (2002), por ejemplo, indica que a menudo Dewey expresó una actitud juguetona, bastante rortiana, hacia toda la práctica de la metafísica. Por ejemplo, Brand Blanshard (1980: 45-46), alumno de Dewey al final de la década de los años veinte, describe vívidamente cómo Dewey utilizó una vez una metáfora del fútbol cuando discutían la naturaleza de la metafísica filosófica. Al ser confrontado por un estudiante que sostenía que metafísicos serios, como Enmanuel Kant, debieron seguramente haber tenido motivos puramente teóricos, de ninguna manera prácticos, para realizar sus estudios metafísicos, Dewey respondió que al igual que en el fútbol, el interés real de un jugador no es simplemente entregar el balón en un lugar determinado, sino hacerlo siguiendo las reglas del juego; en contra de la respectiva oposición, Kant, también, trató de ser tan exitoso como le fuera posible en el juego de la metafísica, en el que uno debe superar a sus oponentes de acuerdo con las normas del análisis lógico -siendo más sutil y congruente que sus rivales. ${ }^{8}$

¿De dónde provienen entonces las reglas de estos juegos? Aquí se rompe la analogía con el fútbol, pues en el caso de los juegos oficiales y deportes como el fútbol, las reglas no se crean y se mantienen en el proceso de la práctica del juego en sí -en lugar de eso, ha existo un grupo de personas que establecen las reglas y las legitimizan como oficiales. Esto no es el caso de las prácticas científicas, filosóficas y otras actividades académicas. Con relación a la reciente discusión sobre los méritos de las ideas de Steve Fuller y Joseph Rouse (Remedios, 2003;

8 Blanshard (1980 : 46), comprometido con el racionalismo toda su vida, concluye, "No me convenció, pero la analogía es lo suficientemente fuerte como para hacer una pausa”. 
Rouse 2003; Fuller, 2003), estamos más en consonancia con las ideas de Rouse $(2003,467)$ quien afirma que es son las prácticas científicas continuas en sí las que interpretan lo que son las prácticas y quién o qué les pertenece. Fuller quiere más. Aunque es suficientemente cuidadoso al plantear que su "concepción universalista de la ciencia" de ninguna manera se compromete con "alguna ofensa metafísica cerrada" -también conocida como "realismo" o "esencialismo"-, y afirma ser "incluso más no representacionalista que Rouse", continúa, sin embargo, en la búsqueda de algún "ideal científico" (Fuller, 2003 : 472, 476). Fuller rechaza la definición de ciencia de Rouse en términos de una comunidad de personas que interactúan para producir conocimientos, pues "esto hace de la ciencia un gremio, cuyos fallos no tienen un tribunal de apelaciones más alto" (Fuller, 2003 : 475). Fuller cree, evidentemente, haber encontrado dicho tribunal de apelaciones en su epistemología social filosófica, cuyo objetivo es "la división óptima del trabajo cognitivo" (Fuller, $1988: 3$ ).

La sociologización de la filosofía significa, entre otras cosas, que no puede haber ningún otro "tribunal" para lo que es científicamente correcto que la comunidad científica pertinente. De hecho, "una ciencia crea y destruye sus propios criterios como resultado de sus propias operaciones, no las de la filosofía" (Fuchs, $2001: 85$ ). Tampoco existe ningún otro criterio para la grandeza o la creatividad de un filósofo que el respeto de sus pares. Ser un gran filósofo simplemente debe ser discutido por la comunidad intelectual, debe ser citado por generación tras generación de pensadores y ser mencionado en los libros de historia (Collins, 1998 : 30-31, 58-61). ${ }^{9}$ De hecho, para los pragmáticos saber significa sencillamente hacer y los hechos pertinentes para los intelectuales en la fase de informar o articular el conocimiento proposicional están relacionados con el logro en la publicación de artículos, la argumentación, la consecución de posiciones y confianza entre colegas, etc. Esto es más evidente en el caso de los filósofos quienes, a diferencia de los científicos, no pasan por una fase de actividades de indagación no lingüística antes de la fase de articulación de las propuestas. Wilfrid Sellars, cuyo nominalismo psicológico "según el cual toda conciencia [...] es un asunto lingüístico", lo expresa bien: "en la caracterización de un episodio o un estado como el del conocimiento [...] estamos colocándolo en el espacio lógico de las razones, de justificar y poder justificar lo que uno dice" (Sellars 1956 : §§ 29, 36).

9 Evaluar el estatus histórico de un intelectual y, por lo tanto, es claro que predecir además qué predicciones sobre dicho estatus resultaron ser correctas requiere una cantidad de tiempo considerable. Se necesitan por lo menos dos o tres generaciones de investigadores para empezar a obtener respuestas confiables a la cuestión de cuáles de los pensadores actuales pasarán a la historia como líderes intelectuales (Collins, 1998 : 58-61, 620-21). 
Las ideas son completamente sociales, lo cual es más transparente precisamente en las comunidades de intelectuales, pues para que sus ideas sean reconocidas como buenas -o quizás verdaderamente como "ideas"- deben reconocerse en la conversación constante de la comunidad respectiva - para ser importantes, para lograr cierto éxito (Collins, 1998 : 31). Esto está estrechamente relacionado con un interesante campo de investigación denominado sociología de la mente (Collins 1989; Coulter 1979; Mead, [1913] 2005), en el cual no podemos profundizar debido al poco espacio de este artículo, pero que consideramos es perfectamente acorde con las directrices metodológicas que desarrollamos con el nombre de sociologización de la filosofía. La idea básica es que uno sólo tiene conciencia sobre sus pensamientos cuando se los comunica a uno mismo o a otros, en un sentido fuerte, no se puede pensar independientemente de una comunidad que utiliza el lenguaje.

\section{La sociología relacional de Bourdieu}

A nuestro enfoque Deweyano de sociologización lo hemos denominado relacionalismo metodológico. El mismo término fue utilizado por Pierre Bourdieu para describir su posición (Bourdieu y Wacquant, 1992 : 15). Además, "el cuerpo socializado" y los conceptos relacionados de Bourdieu (1992) podrían recordarnos el pragmatismo de Dewey y la evasión del dualismo sujeto-objeto (Kauppi, 2000 : 52). Por otra parte, Bourdieu (1988: xi) es conocido por intentar "atrapar al Homo Academicus, clasificador supremo entre los clasificadores, en la red de sus propias clasificaciones", es decir, por intentar sociologizar una variedad de campos académicos, incluyendo la filosofía (Bourdieu, 2000). Sin embargo, también existen ciertos factores cruciales que el pensamiento de Bourdieu comparte con la filosofización de la sociología, por ejemplo: sus intentos por esbozar una teoría de la práctica (Bourdieu, 1977).

Debido a la influencia de la tradición estructuralista, el relacionalismo de Bourdieu (1998 : 3) culmina en afirmaciones como "lo real es relacional" -toda distinción, toda diferencia es "una propiedad relacional existente solamente a través de su relación con otras propiedades". A pesar de que Bourdieu (1998: 3) advierte contra una interpretación sustancialista, esencialista e "ingenuamente realista" de su análisis, también está dispuesto a identificarse como realista, sosteniendo incluso que "la representación realista de la acción humana [...] es la primera condición para el conocimiento científico del mundo social” (Bourdieu, 1998 : viii-ix). De hecho, en la esencia del relacionalismo metodológico de Bourdieu está la idea de que "el centro de la realidad social [...] radica en las relaciones" (Bourdieu y Wacquant, 1992 : 15). Bourdieu es suficientemente 
anti-representacionalista para evitar los problemas de una sencilla teoría de correspondencia de la verdad, pero, como señala Jeffrey Alexander (1995), meta-teóricamente Bourdieu sigue siendo un realista en búsqueda de la "verdad verdadera”. Por consiguiente, afín a los realistas pragmáticos, Bourdieu evita los errores de las formas más ingenuas del esencialismo, pero aún así se une a la tarea de revelar la realidad. Aunque Bourdieu también ha hecho algunas referencias favorables al pragmatismo de Dewey (Bourdieu y Wacquant, 1992 : 122), de un modo completamente no pragmatista fortalece repetidamente sus descripciones sociológicas con expresiones como "relaciones objetivas", "estructuras objetivas", y "orden objetivo", mientras curiosamente deja abierto lo que quiere decir exactamente con el término objetivo (Bourdieu, 1998 : vii, 12, 26).

Adoptar el relacionalismo metodológico pragmático desarrollado en este documento significa reemplazar la idea de la investigación, en la que se revelan las estructuras de la realidad, por una noción de investigación en la que simplemente se organizan datos en redes coherentes de descripciones útiles. El conocimiento científico no se desarrolla mediante una profundización vertical, sino mediante una expansión horizontal (Rorty 1999 : 82-83; Kivinen y Ristelä, 2002 : 424). Mientras que Bourdieu, por ejemplo, afirma que sus conceptos claves, "habitus" y "campo", son relacionales (Bourdieu y Wacquant 1992: 16), nos gustaría anotar que en realidad todo concepto y, por lo tanto, también todo conocimiento es relacional: es imposible comprender algo que sea "no relacional". Los atributos -descritos de alguna manera- son lo que son a través de las relaciones que tienen que otros atributos -descritos de alguna manera-, pero ni los atributos, ni sus interrelaciones, pueden ser tratados cognitivamente hasta que se describan por medio de símbolos en algún juego de lenguaje.

El mismo Bourdieu ha observado algún paralelo cercano entre su concepto de habitus y el concepto de "hábito" de Dewey (Bourdieu y Wacquant, 1992 : 122-23 y n. 77). Existen, de hecho, algunas similitudes entre las posiciones de Bourdieu y de Dewey ilustradas por estos conceptos, pero también existen algunas diferencias importantes. Las similitudes tienen que ver con el reconocimiento compartido por parte de Bourdieu y Dewey de las actividades humanas corporeizadas, no conscientes, mientras las diferencias giran en torno a las tendencias estructuralistas filosofizantes de Bourdieu, que, debido a su tono cosificante, van en contra del enfoque totalmente pragmático de Dewey.

A diferencia de Jeffrey Alexander (1995: 152), quien ironiza la actividad no consciente presente en el concepto de habitus de Bourdieu con el título de "El oxímoron de la 'Estrategia inconsciente", encontramos que las ideas de Bourdieu sobre "la sensación del de juego" y "el sentido práctico", que se 
originaron en el pensamiento de Maurice Merleau- Ponty, son muy oportunas en muchos casos. Estos conceptos son herramientas útiles para describir a los actores de un campo académico, en el mismo sentido que se pueden utilizar para describir, digamos, a los buenos jugadores de baloncesto, quienes incluso en el momento más apasionado del juego intuyen los movimientos de sus compańeros de equipo y sus oponentes. La variedad de situaciones pertinentes se convierte en un sedimento, por decirlo así, en el cuerpo, de tal manera que formas pre-programadas de actuar estarán a la espera de ser reactivadas (Bourdieu y Wacquant, 1992 : 20-22). Considerando que un mal jugador -tanto en un campo académico como en un juego de baloncesto- se mueve de forma asincrónica demasiado pronto o demasiado tarde, un buen jugador se adelanta al juego. Sólo un jugador habilidoso que ha interiorizado la dirección del juego, puede integrarse con el juego. Un buen sentido del juego hace posible adaptarse a las necesidades del mundo académico y, por lo tanto, tener una exitosa carrera académica, sin fijárselo conscientemente como una meta -habiéndose propuesto jugar juegos académicos. El sentido del juego científico permite hacer las cosas correctas en el momento oportuno sin plantearse conscientemente qué hacer (Bourdieu, 1998 : 25, 79-88; Collins, $1998: 33$, 38-40, 48 y 49). De hecho, al igual que la investigación científica se asemeja de muchas formas a ejercer un oficio, los investigadores son como cualquier otro trabajador, en el sentido que hacen lo que hacen en gran medida por la fuerza corporeizada de cómo hacerlo. Las habilidades que hacen posible la investigación "no provienen de libros de texto o teorías, sino de la socialización en una práctica transmitida a través de lazos generacionales entre maestros y aprendices" (Fuchs, 2001 : 68). Esta es la primera superficie de contacto entre Bourdieu y el pragmatismo: ambos están centrados profundamente en la acción en su concepción de los seres humanos.

Si el concepto de habitus siguiere uniformemente estas líneas de pensamiento, a fin de ser concebido como una forma centrada en la acción de describir el sentido práctico, corporeizado de lo que hay que hacer en una situación dada, manifestaría las ideas pragmáticas más recomendables en el trabajo de Bourdieu. Lamentablemente, sin embargo, el uso que Bourdieu hace del término es bastante ambiguo. Como primera medida, normalmente deja abierta la posibilidad de si el habitus es algún tipo de sujeto actuante en sí o un principio clasificatorio con el que los investigadores explican las acciones (Bourdieu, $1998: 8$ ). Por otra parte, aunque Dewey considera que los hábitos son características de los individuos, las cuales se diferencian lacónicamente de las costumbres colectivas, el habitus de Bourdieu también es ambiguo con respecto a si es una característica de las personas o de los colectivos. Algunas veces la terminología de Bourdieu parece 
ser casi intencionalmente críptica y mistificante, pues describe el habitus como lo "colectivo individualizado" y lo "individual colectivizado" -como algo que es "un operador [...] de racionalidad práctica inmanente en un sistema histórico de relaciones social y que, por lo tanto, es trascendental para el individuo" y "un mecanismo de estructuración que funciona desde dentro de los agentes"que, aún así, no es "ni estrictamente individual ni plenamente determinante de la conducta" (Bourdieu y Wacquant, 1992 : 18-19). En vista de esto, parece que hay mucho por ganar si se reemplazan estos laberintos conceptuales con el concepto de "hábito" de Dewey. En lugar de un habitus integrador, podemos hablar de muchos hábitos diferentes, algunos de los cuales se concretan en un tipo de acciones, en un tipo de circunstancias, y otros en otros tipos.

El concepto de hábito de Dewey no requiere explicaciones estructurales profundas; un hábito es simplemente un hábito (Valverde, 1998 : 220-21; Hodgson, 2004 : 187, n. 8). El equivalente más cercano del concepto de habitus de Bourdieu en el pensamiento de Dewey es probablemente el concepto de carácter, que nuevamente no es nada más que una "interpenetración de hábitos" (Dewey, [1922] 1983 : 29 y 30; Kivinen, 2002 :198; Kivinen y Ristelä, 2002 : 421-23). Desde la perspectiva de Dewey, los hábitos son exigencias de la actividad que constituyen al ser y la voluntad humana. "En cualquier sentido inteligible de la palabra voluntad, ellos son voluntad" (Dewey, [1922] 1983 : 21; Dennett, 2003). En consecuencia, no hay necesidad de plantear el concepto de habitus con algún poder integrado específico, no mayor que el alma o su equivalente moderno, el centro cognitivo o los niveles de cognición o meta-cognición. Basta con comprender toda la gama de actividades humanas como hábito dependiente (Kivinen y Ristelä, 2003). Así, evitamos complicaciones innecesarias tales como la idea de reglas inmanentes e inconscientes que las personas necesitan seguir en sus prácticas cotidianas:

Las reglas inmanentes son simplemente una metáfora para todo tipo de formas de hacer las cosas. Luego, aparece el psicólogo o el sociólogo y escribe las normas como reglas. No existen reglas inconscientes, los hábitos son sólo hábitos (Harré 2002, 117; Pleasants, 1996).

Reconocer que nuestras actividades dependientes de los hábitos a menudo son también institucionalizadas como costumbres sociales, de ninguna manera requiere plantear "lazos" específicos entre los individuos y las estructuras. El concepto de hábito cumple todas las tareas necesarias que el concepto súperindividual de habitus de Bourdieu, sin sus problemas; los únicos lazos necesarios son los 
hábitos de las personas que se conectan con los hábitos de los demás. Como lo expresa Dewey (1983 [1922] : 19), "nuestros hábitos individuales son lazos en la formación de la cadena sin fin de la humanidad". En contraste, el habitus de Bourdieu (2000 : 157) como una especie de centro neurálgico colectivo/personal capaz de hacer que las cosas ocurran en el mundo social, tiene aparentemente como fin ser un enlace entre las personas y las estructuras. Por ejemplo, Garry Potter (2000 : 237), un declarado realista crítico y alma gemela de Bhaskar, considera que el habitus de Bourdieu puede perfectamente ser planteado para ligar la teoría de la estructura y una teoría de la acción. En su ensayo "A favor de Bourdieu, en contra de Alexander", Potter (2000) interpreta las perspectivas de Bourdieu como compatibles con su propia ontología social, la cual abarca muchos niveles diferentes de la realidad social. ${ }^{10}$ De acuerdo con esta interpretación, en principio no existe ninguna contradicción entre la propuesta de realismo crítico de Bhaskar y los análisis de Bourdieu. Como sugiere Potter, la concepción de la estructura social de Bourdieu podría en realidad ser metafísico realista (Potter, 2000 : 245-46; Vandenberghe, 1999); y, de hecho, esto toma un inconfundible tono realista cuando Bourdieu define como tarea de la sociología "descubrir las estructuras más profundamente enterradas de los diferentes mundos sociales" (Bourdieu, 1996 : 1; Bourdieu y Wacquant, 1992 : 7).

Existen indicios de afinidad entre la idea realista de estructuras metafísicas con el concepto de campo de Bourdieu, pues éste afirma que un campo está compuesto por relaciones objetivas entre posiciones definidas objetivamente, siendo "un sistema con patrones de fuerzas objetivas - de igual manera que un campo magnético-" (Bourdieu y Wacquant, 1992 : 16-17, 97; Martin, 2003). No es difícil encontrar en este caso otro punto de contacto con Bhaskar (1979 : 15-16; 2002 : 97), quien ha explotado la analogía del campo magnético para abogar por una ontología de las estructuras sociales como entidades. Después de todo, parece que Bhaskar opinara que el acuerdo intuitivo sobre la realidad de los campos magnéticos podría convencer a su audiencia a que admitiera la realidad de las estructuras sociales. Sin embargo, aun cuando el concepto de campo pueda ser una herramienta viable para comprender y explicar las acciones sociales, la analogía entre el campo social y el campo magnético es extremadamente engańosa. Mientras que los efectos de los campos magnéticos son bastante predecibles -el concepto de campo magnético es útil en los juegos de lenguajes cotidianos, en el pensamiento cotidiano y también en la " física popular"- los efectos de los campos sociales no lo son, y se debe tener cuidado con la equi-

10 Si esto es cierto, entonces Bourdieu está entre quienes John Heil (2003 : 205) descaradamente caracteriza como "enamorados de los niveles". 
vocación latente con este término, tanto cuando se utilizan juegos de lenguaje científico social como cuando se presentan los resultados de una investigación científica social ante un público no especializado más amplio.

Si bien Bourdieu es evidentemente un pensador con una orientación pragmática, en el sentido que aprecia las ideas como sentido del juego y saber corporeizado, también es un ejemplo de la filosofización de la sociología o teorizador de la práctica (Bourdieu, 1977 : 2000). Compartimos la visión de que la práctica es fundamental, pero desde nuestro punto de vista pragmático no tiene sentido tratar de construir una teoría de la práctica. Es precisamente debido a lo fundamental de la práctica -debido al hecho de que todo es práctico y sólo puede ser sopesado en la acción- que todas las teorías deben ser concebidas como herramientas para la acción. Como herramientas de un investigador, las teorías son instrumentos que sólo se pueden probar en las prácticas del trabajo de investigación, en los estudios de casos rigurosamente limitados que tienen como fin resolver algunos problemas de investigación específicos -y que al final se espera que contribuyan a la solución de algunos de los problemas reales que las personas enfrentan. Las teorías son buenas siempre y cuando sean herramientas útiles para resolver los problemas de investigación que los investigadores se han planteado. Desde el punto de vista pragmático, tenemos que adoptar la estricta demanda de operacionalización-comprender las teorías en términos de acciones a realizar-y esto significa, entre otras cosas, abandonar la idea de que la creciente complejidad de una teoría y el uso del léxico doctrinal peculiar se pueden justificarse afirmando que son necesarios al captar la complejidad y la indefinición del mundo real (Bourdieu y Wacquant, 1992 : 22-23 y n. 40 y 41). En su lugar, necesitamos un marco de referencias inequívocamente operacionalizable -es decir, una teoría sencilla con $t$ minúscula- que nos sirva como un posible conjunto de herramientas para resolver problemas específicos de investigación.

\section{El relacionalismo metodológico en las ciencias sociales: hacia la investigación operacionalista enfocada en los problemas}

Como lo señaló C. S. Peirce, una creencia es un hábito de acción, y por lo tanto, una verdadera creencia puede ser entendida, sencillamente, como un hábito de acción funcional en la práctica (Peirce, [1878] 1986 : 263-66). Así, el objetivo de cualquier investigación es el acuerdo sobre una opinión, que en el caso de los conocimientos científicos también es una cuestión de lograr la justificación o legitimación de una opinión de parte de la comunidad científica. "Puede parecernos que esto no es suficiente, y que buscamos, no simplemente una opinión, sino una opinión verdadera", Peirce resalta el antiguo argumento 
realista, derivado al menos a partir de Platón, sólo para hacer hincapié en que, de hecho:

[...] tan pronto como se logra una creencia firme, quedamos completamente satisfechos [...] Lo más que puede sostenerse es que buscamos una creencia que pensamos que es cierta, pero creemos que cada una de nuestras creencias es verdadera y, de hecho, afirmarlo es una mera tautología ([1877] $1986: 248)$.

Por lo tanto, aunque Peirce se asemeja a los filósofos realistas más que los otros clásicos del pragmatismo, el punto de vista del actor humano se plantea como inevitable en su perspectiva, al igual que en la de James y Dewey.

Cuando las creencias son entendidas como hábitos de acción, se concreta que se puede pensar en cualquier cosa sólo desde el punto de vista del actor, que nadie puede salirse de sus propias acciones. Por consiguiente, todos los hechos son operativos en el sentido en que están ligados inextricablemente a la acción; los hechos son lo que son con respecto a una u otra operación, desde el punto de vista de uno u otro actor (Peirce, [1877] 1986, 1986 [1878]; James, [1881] 1979 : 99; 1981 : 27ff.; Dewey, [1929] 1984 : 89-92; [1938] 1991, 116-17). Como investigadores humanos, sólo nos interesan las causas relevantes para nuestras actividades -causas lo suficientemente específicas para ser útiles para nuestros propósitos (James, [1880] 1979 : 163-66; Dewey, [1938] 1991 : 52-57). "Esto es $[\ldots]$ la necesidad de imponernos, como seres humanos, limites para nuestra opinión", señala James:

$\mathrm{Al}$ igual que un astrónomo quien al estudiar los movimientos de las mareas en el océano, no tiene en cuenta las olas hechas por el viento [...] Al igual que el francotirador quien, en al cuadrar la mira de su rifle, considera el movimiento del viento, más no [...] el movimiento de la tierra y el sistema solar ([1880] $1979: 166)$.

Las consultas científicas también deben limitarse a estudios de casos específicos que apunten a responder preguntas de investigación planteadas desde el punto de vista de los propios investigadores.

El conocimiento se adquiere haciendo las cosas, y "las ideas son enunciados no de lo que es o ha sido sino de actos por realizar" (Dewey, [1929] 1984 : 111) $y$, por lo tanto, cada vez que se selecciona algo como la causa de algo en una investigación, se hace desde el punto de vista del actor de una investigación. Se trata de una selección que implica distinguir la causa de las condiciones del trasfondo y depende del conocimiento que uno tiene y puede utilizar en las predicciones. 
Por consiguiente, no tiene sentido suponer que las descripciones que utilizamos de alguna manera representan "las causas verdaderas" sin ningún punto de vista (Putnam, 1990 : 87; Rorty, 1999 : 32-33). Entendido de esta manera, dentro del proceso de investigación científica social real no hay espacio para contemplar los elementos que son independientes y preceden a la investigación en el sentido de un metafísico. El avance de la ciencia no se debe entender en términos de la ciencia que se aproxima, cada vez más, a las verdades reales que se pueden determinar metafísicamente, sino, como anota Rorty (1998 : 5), en términos de la facultad creciente de hacer predicciones y ayudar a las personas a controlar una diversidad de situaciones de una mejor manera que antes.

No obstante, en lo que Rorty no enfatiza suficientemente es en que el creciente poder de hacer predicciones requiere un trabajo metodológico notable. Cuando pensamos en el saber en términos de acciones y concebimos toda la conceptualización científica como herramientas para las acciones, vemos claramente que, al igual que cualquier otra herramienta que un científico podría utilizar-hojas de cálculo, radiotelescopios o microscopios electrónicos- también las herramientas conceptuales, como el "relacionalismo", necesitan un mantenimiento continuo, y eso es lo que tratamos ofrecer en este artículo. Un científico social que quiera estar actualizado necesita estudios metodológicos a través de los cuales refine constantemente el conjunto de herramientas conceptuales -asegurándose que los conceptos continúen siendo fácilmente operacionalizables con relación a la solución de los problemas de la investigación científica social más comunes dentro del marco de referencia pertinente. Por lo tanto, se puede anotar que aunque el contraste entre Rorty y Searle, examinado anteriormente, capta bien la distinción entre la sociologización de la filosofía y la filosofización de la sociología, existen otros aspectos de nuestro relacionalismo metodológico que nos ubican entre las posiciones de Rorty y Searle. En estos aspectos, nos apoyamos directamente en los clásicos del pragmatismo, en particular en Dewey, destacando que, al ser entendida correctamente, "la filosofía de las ciencias sociales" se concentra especialmente en el trabajo metodológico en el ámbito de las ciencias sociales.

Siguiendo el enfoque operacionalista de Dewey, llegamos a una forma práctica, de comprender las ciencias sociales orientada en los problemas. Debido a que todo conocimiento humano está relacionado con los objetivos del investigador y todas las creencias deben ser sopesadas sólo en la acción intencional y en sus consecuencias, también las conceptualizaciones científicas sociales utilizadas por los investigadores son herramientas que deben ser operacionalizadas a las cosas por hacer. La validez de cualquier creencia sólo se puede probar tratando de actuar consecuentemente (Dewey [1929] 1984 : 103, 157, 242-43). Por lo tanto, con 
el objetivo de averiguar qué conflictos y cooperación reales se presentan entre las personas en una comunidad específica y qué problemas sociales específicos enfrentan estas personas en su vida cotidiana, los científicos pragmáticos sociales confrontan la cuestión de cómo operacionalizar los problemas de investigación más comunes en formas que sean mesurables para que posteriormente se puedan especificar las causas y consecuencias relevantes para las preguntas que nos ocupan (Kivinen y Piiroinen, 2004; Dewey, [1938] 1991 : 481-505; Dewey, [1920] 1982 : 187-94; James, [1880] 1979: 164-66, 170-76; [1890] 1979, 193-94).

Tiene el mismo interés estudiar las esencias metafísicas de las herramientas conceptuales -por ejemplo "las estructuras sociales" o "las creencias racionales"como estudiar la esencia metafísica de tenedores y cucharas -usando el ejemplo de Louis Menand (2001: 361):

Cuando un tenedor resulta inadecuado para la tarea de tomarse la sopa, no tiene sentido discutir acerca de si hay algo inherente a la naturaleza de los tenedores o de la sopa que explique el fracaso. Simplemente se busca una cuchara.

Los relacionalistas metodológicos evitan todo el juego de lenguaje ontológico y, con ello, las dos puntas del debate ontológico sobre el individualismo / colectivismo. Como los conceptos utilizados en las ciencias sociales son entendidos simplemente como herramientas más o menos útiles para describir y predecir las actividades humanas -operacionalizables para actividades específicas de investigación que un investigador puede llevar a cabo- no tenemos necesidad de hipostasiar sobre los "referentes" de estos conceptos y mucho menos afirmar que algunos de dichos referentes deban ser planteados como un fundamento filosófico a priori de la investigación científica social. Ninguno de los mecanismos descriptivos para clasificar las actividades debe ser considerado indispensable en el sentido que las actividades de investigación científica social no podrían ser iniciadas o desarrolladas sin ellos.

Las conceptualizaciones que hemos encontrado más adecuadas para describir las actividades humanas se encuentran entre las herramientas más tradicionales del pragmatismo que son acordes con lo que podríamos denominar la historia darwiniana, la cual describe la vida como transacciones de organismos y medio ambiente, como acción e interacción. Como lo expresa Dewey (1983 [1922]), los seres humanos se pueden describir como organismos que operan simplemente sobre la fortaleza de sus hábitos. Un organismo viviente, como un ser humano, continuamente actúa en virtud de sus hábitos, lidiando con el medio durante toda su vida, sin necesitar razones concretas para ello; no tiene sentido pregun- 
tarse por qué vivir y actuar, sino cómo. Así podemos entender claramente que las personas logran hacer las cosas cotidianas correctamente sin pensar en ellas conscientemente -saben cómo hacer las cosas, mucho más que las cosas que pueden explicar (Ryle, 1949 : 25-61; Polanyi, 1969).

A medida que las interacciones entre las personas se institucionalizan en el tiempo y adquieren formas comunales establecidas, surgen las costumbres y las culturas. Las comunidades, entonces, pueden considerarse como conjuntos de costumbres institucionalizadas, heredadas de una generación a otra y de un individuo a otro. En toda comunidad humana, el lenguaje juega un papel crucial y por eso, siempre habrá el saber-“cómo hacer" corporeizado y los conocimientos lingüísticos, que se distinguen conceptualmente el uno del otro. De hecho, ya un cuarto de siglo antes de Gilbert Ryle, Dewey (1983 [1922] : 124-25) distinguió el saber proposicional acerca de algo, de los saberes corporeizados del saber-cómo, indicando que sólo el primero es "conocimiento que implica una reflexión y un reconocimiento conscientes", y por lo tanto, debe ser contrastado con toda "habilidad práctica adquirida [que] no es conocimiento, excepto por cortesía". ${ }^{11}$

El concepto de hábito sigue siendo el concepto clave en la metodología pragmática: los filósofos, al igual que los pianistas, carpinteros, médicos y políticos son capaces de hacer lo que hacen por llevar los hábitos corporeizados que llevan. "El acto debe preceder al pensamiento y un hábito a la capacidad de evocar el pensamiento a voluntad" (Dewey [1922] 1983 : 25, 128). Los investigadores científicos sociales están interesados en la vida social de una u otra comunidad, y debido a que las prácticas humanas dentro de las comunidades son en su mayor parte acciones colectivas sobre la fortaleza del saber-cómo, el tema de las investigaciones científicas sociales no se puede restringir al lenguaje y a la conversación. Sin embargo, el conocimiento adquirido en cualquier campo científico, el conocimiento valorado en las conversaciones de expertos y registrado en libros y bases de datos, es sólo saber lingüístico acerca de algo -que, por supuesto, sigue estando ligado y orientado en hacer las cosas. El conocimiento lingüístico acerca de algo es la única forma de conocimiento que podemos explicar, discutir y debatir y la única con la que podemos esperar mejorar nuestra comunicación y coordinación de acciones en cualquier comunidad. Por lo tanto, el desarrollo de la metodología en un campo de investigación se fortalecerá significativamente si se tiene en cuenta la sociología de dicho campo.

11 Es precisamente debido a esta distinción conceptual entre conocimiento proposicional y destrezas para el saber-cómo, y enfatizando (Archer, 2000 : 164) que sólo el primero es denominado correctamente conocimiento, que podemos evitar las confusiones involucradas al hablar acerca de un conocimiento no lingüístico comprendido directamente (Kivinen y Piiroinen, 2006). 
Como lo entiende Dewey, el razonamiento científico se constituye en gran medida en las conversaciones que desarrollan y definen creencias -como hábitos de acción- con relación a otras creencias. Dentro de esta conversación, los conceptos se transforman en algo cada más relevante para el problema que se estudia, esperando que termine sugiriendo operaciones de investigación reales para probar la aplicabilidad de dichas creencias (Dewey [1938] 1991 : 115). De acuerdo a este planteamiento de Dewey sobre la conclusión de que la investigación científica social debe ser vista como una forma de tratar de responder cuestiones de investigación importantes de manera práctica, que ayuden a la solución de problemas reales de las personas en su vida social, los científicos sociales pragmáticos consideran que no tiene ningún sentido teorizar sobre la "verdadera naturaleza de la realidad social".

\section{Palabras finales}

Hemos contrastado dos formas opuestas de comprender el relacionalismo y la investigación en las ciencias sociales -la filosofización de la sociología y la sociologización de la filosofía. Como pragmáticos, preferimos sociologizar la filosofía, comprendiendo todas las teorías y las doctrinas en contextos sociales e históricos adecuados. A diferencia de quienes consideran que las revelaciones filosóficas de la realidad constituyen la base adecuada para las prácticas de la investigación científica, nosotros preferimos enfocar las ciencias sociales desde la dirección contraria - desde la perspectiva de la metodología basada en el problema, en vez de la teorización metafísica. Abandonamos el dualismo filosófico entre sujeto cognoscente y objetos por conocer, un dualismo que lleva a los realistas a pensar que es necesario fundamentar las investigaciones científicas en la teorización, en primer lugar, sobre la naturaleza final de los objetos a conocer -ontología- y, en segundo lugar, sobre de la relación entre el sujeto y los objetos -epistemología. Nuestro propio enfoque, denominado relacionalismo metodológico, toma de los clásicos del pragmatismo la enseñanza que la filosofía de las ciencias sociales es más adecuada para la labor metodológica dentro del campo de ciencias sociales. Así, nos diferenciamos tanto de los realistas que hacen énfasis en el papel de la fundamentación ontológica a expensas de la metodología y de los relativistas extremos que no ven qué caso tiene la labor metodológica que sugerimos. Además, nuestro relacionalismo metodológico difiere del enfoque de Pierre Bourdieu -con el mismo nombre- por ser más fiel a la idea de que la práctica es fundamental, pero evitando el atractivo de construir una teoría de la práctica.

Durante su larga carrera, Dewey criticó a los letrados que pensaban que el conocimiento es válido científicamente sólo en la medida en que revele la reali- 
dad como tal. Le pareció más importante preguntarse cómo diferentes tipos de concepciones podrían ayudar a las personas a solucionar los problemas prácticos que enfrentan. La certidumbre cognitiva está integrada a la necesidad de garantizar los resultados de las acciones, no con eternas verdades metafísicas (Dewey, [1929] 1984 : 30-31, 36). Abandonar inalcanzables requisitos metafísicos previos ayudará a avanzar hacia enfoques totalmente operacionalistas pragmáticos centrados en la acción, los cuales apoyan los intentos de los científicos sociales por ayudar a resolver problemas de la vida social a través de estudios de casos orientados en los problemas.

\section{Referencias}

Alexander, J. 1995. Fin de siècle social theory: Relativism, reduction, and the problem of reason. London: Verso.

Archer, M. S. 1995. Realist social theory: The morphogenetic approach. Cambridge: Cambridge University Press.

bridge University Press. 2000. Being human: The problem of agency. Cambridge: Cam-

Bello, D. C., and C. Chelariu, and L. Zhang. 2003. The antecedents and performance consequences of relacionalismo in export distribution channels. Journal of Business Research 56 (1): 1-16.

Bhaskar, R. 1979. The possibility of naturalism: A philosophical critique of the contemporary human sciences. Brighton: Harvester.

. 1986. Scientific realism and human emancipation. London: Verso. 2002. From science to emancipation: Alienation and the actuality of enlightenment. New Delhi: Sage.

Blanshard, B. 1980. Autobiography. In The library of living philosophers, vol. 15, edited by P. A. Schilpp. La Salle, IL: Open Court.

Bourdieu, P. 1977. Outline of a theory of practice. Cambridge: Cambridge University Press.

1988. Homo academicus. Cambridge: Polity.

1992. The logic of practice. Cambridge: Polity.

1993. The field of cultural production: Essays on art and literature.

Cambridge: Polity. 
bridge: Polity.

1996. The state nobility: Elite schools in the field of power. Cam-

1998. Practical reason: On the theory of action. Cambridge: Polity. 2000. Pascalian meditations. Cambridge: Polity.

Bourdieu, P., and L. Wacquant. 1992. An invitation to reflexive sociology. Chicago: University of Chicago Press.

Cambridge Dictionary of Philosophy. 1999. General editor R. Audi. 2nd ed. Cambridge: Cambridge University Press.

Collins, R. 1989. Toward a neo-Meadian sociology of mind. Symbolic Interaction 12 (1): 1-32.

1998. The sociology of philosophies: A global theory of intellectual change. Cambridge, MA: Belknap Press of Harvard University Press.

Coulter, J. 1979. The social construction of mind: Studies in ethnomethodology and linguistic philosophy. London: Macmillan.

Kivinen, Piiroinen / Toward Pragmatist Methodological Relacionalismo 23

Davidson, D. 1984. On the very idea of a conceptual scheme. In his Inquiries into truth and interpretation. Oxford: Clarendon.

. 1990. The structure and content of truth. Journal of Philosophy

87 (6): 279-328.

Dennett, D. C. 2003. Freedom evolves. New York: Penguin.

Dewey, J. [1920] 1982. Reconstruction in philosophy and essays. In The middle works of John

Dewey, vol. 12, edited by J. A. Boydston. Carbondale: Southern Illinois University Press.

[1922] 1983. Human nature and conduct. In The middle works

of John Dewey, vol. 14, edited by J. A. Boydston. Carbondale: Southern Illinois University Press.

[1925] 1981. Experience and nature. In The later works of John Dewey, vol. 1, edited by J. A. Boydston. Carbondale: Southern Illinois University Press.

[1929] 1984. The quest for certainty. In The later works of John Dewey, vol. 4, edited by J. A. Boydston. Carbondale: Southern Illinois University Press. 
. [1938] 1991. Logic: The theory of inquiry. In The later works of John Dewey, vol. 12, edited by J. A. Boydston. Carbondale: Southern Illinois University Press.

Elias, N. 1976. Über den prozeß der zivilisation: Soziogenetische und psychogenetische untersuchungen.

Erster Band: Wandlungen des verhaltens in den weltlichen oberschichten des abendlandes. Taschenbuch. Frankfurt am Main: Suhrkamp.

1982. Über den prozeß der zivilisation: Soziogenetische und psychogenetische untersuchungen. Zweiter Band: Wandlungen der gesellschaft entwurf zu einer theorie der zivilisation. Taschenbuch. Frankfurt am Main: Suhrkamp.

Emirbayer, M. 1997. Manifesto for a relational sociology. American Journal of Sociology 103 (2): 281-317.

Fuchs, S. 2001. Against essentialism: A theory of culture and society. Cambridge, MA: Harvard University Press.

Fuller, S. 1988. Social epistemology. Bloomington: Indiana University Press. 2003. The unended quest for legitimacy in science. Philosophy of the Social Sciences 33 (4): 472-78.

Haack, S. 1993. Evidence and inquiry: Towards reconstruction in epistemology. Oxford: Blackwell.

1998. Manifesto of a passionate moderate: Unfashionable essays. Chicago: University of Chicago Press.

Harré, R. 1986. Varieties of realism: A rationale for the natural sciences. Oxford: Blackwell. . 1997. Forward to Aristotle: The case for a hybrid ontology. Journal for the Theory of Social Behaviour 27 (2/3): 173-91.

. 1998. When the knower is also the known. In Knowing the social world, edited by T. May and M. Williams. Buckingham, UK: Open University Press.

on pp. 96-124.]

2002. [Harré's contribution to the discussion in Bhaskar 2002,

Heil, J. 2003. Levels of reality. Ratio 16 (3): 205-21.

Hodgson, G. M. 2004. The evolution of institutional economics: Agency, structure and Darwinism in American institutionalism. London: Routledge. 
Jackson, P., and D. Nexon. 1999. Relations before states: Substance, process and the study of world politics. European Journal of International Relations 5 (3): 291-332.

James, W. [1880] 1979a. Great men and their environment. In The will to believe and other essays in popular philosophy: The works of William James, edited by F. H. Burkhardt. Cambridge, MA: Harvard University Press.

. [1881] 1979b. Reflex action and theism. In The will to believe and other essays in popular philosophy: The works of William James, edited by $\mathrm{F}$. H. Burkhardt. Cambridge, MA: Harvard University Press. 24 Philosophy of the Social Sciences

. [1890] 1979c. The importance of individuals. In The will to believe and other essays in popular philosophy: The works of William James, edited by F. H. Burkhardt. Cambridge, MA: Harvard University Press.

Kauppi, N. 2000. The politics of embodiment: Habits, power, and Pierre Bourdieu's theory. Frankfurt am Main: Peter Lang.

Kiser, E., and M. Hechter. 1991. The role of general theory in comparativehistorical sociology. American Journal of Sociology 97 (1): 1-30.

Kivinen, O. 2002. Higher learning in an age of uncertainty: From postmodern critique to appropriate university practices. In Higher education in a globalising world, edited by J. Enders and O. Fulton. Dordrecht: Kluwer Academic.

Kivinen, O., and T. Piiroinen. 2004. The relevance of ontological commitments in social sciences: Realist and pragmatist viewpoints. Journal for the Theory of Social Behaviour 34 (3): 231-48.

2006. On the limits of a realist conception of knowledge: A pragmatist critique of Archerian realism. Sociological Review 54 (2): 224-41.

Kivinen, O., and P. Ristelä. 2001. Pragmatistisia näkökulmia tiedonhankintaan ja toimintaan: Metodologinen relacionalismoi käytännön toiminnassa [Pragmatist perspectives on the procurement of information and action: Methodological relacionalismo in concrete action].

Sosiologia 38 (4): 249-59.

2002. Even higher learning takes place by doing: From postmodern critique to pragmatic action. Studies in Higher Education 27 (4):419-30. .2003. From constructivism to a pragmatist conception of learning. Oxford Review of Education 29 (3): 363-75. 
Margolis, J. 2002. Dewey's and Rorty's opposed pragmatisms. Transactions of the Charles S. Peirce Society 38 (1/2): 117-35.

2003. The benign antinomy of a constructive realism. In Pragmatic naturalism and realism, edited by J. Shook. Amherst, NY: Prometheus.

Martin, J. L. 2003. What is field theory? American Journal of Sociology 109 (1): $1-34$.

Mayr, E. 1982. The growth of biological thought: Diversity, evolution, and inheritance. Cambridge, MA: Belknap Press of Harvard University Press.

Mead, G. H. [1913] 2005. The social self. In Pragmatism: Critical concepts in philosophy vol. 1, edited by R. B. Goodman. New York: Routledge.

Medina, J. 2003. Wittgenstein and nonsense: Psychologism, Kantianism, and the habitus. International Journal of Philosophical Studies 11 (3): 293-318.

. 2004. In defense of pragmatic contextualism: Wittgenstein and Dewey on meaning and agreement. Philosophical Forum 35 (3): 341-69.

Menand, L. 2001. The metaphysical club. London: HarperCollins.

Oxford Dictionary of Philosophy. 1996. Edited by Simon Blackburn. Oxford: Oxford University Press.

Peirce, C. S. [1877] 1986a. The fixation of belief. In Writings of Charles S. Peirce: A chronological edition, vol. 3, edited by C. J. W. Kloesel. Bloomington: Indiana University Press.

[1878] 1986b. How to make our ideas clear. In Writings of Charles S. Peirce: A chronological edition, vol. 3, edited by C. J. W. Kloesel. Bloomington: Indiana University Press.

Pleasants, N. 1996. Nothing is concealed: De-centring tacit knowledge and rules from social theory. Journal for the Theory of Social Behaviour 26 (3): 233-55.

Polanyi, M. 1969. Personal knowledge: Towards a post-critical philosophy. London: Routledge.

Kivinen, Piiroinen / Toward Pragmatist Methodological Relacionalismo 25

Potter, G. 2000. For Bourdieu, against Alexander: Reality and reduction. Journal for the Theory of Social Behaviour 30 (2): 229-46.

Putnam, H. 1987. The many faces of realism. La Salle, IL: Open Court.

University Press. 1990. Realism with a human face. Cambridge, MA: Harvard 
Remedios, F. 2003. Fuller and Rouse on the legitimation of scientific knowledge. Philosophy of the Social Sciences 33 (4): 444-63.

Rescher, N. 1996. Process metaphysics: An introduction to process philosophy. Albany: State University of New York Press.

2000. Realistic pragmatism: An introduction to pragmatic philosophy. Albany: State University of New York Press.

Rorty, R. 1997. Realism, antirealism, and pragmatism: Comments on Alston, Chisholm, Davidson, Harman, and Searle. In Realism/antirealism and epistemology, edited by C. B. Kulp. Lanham, MD: Rowman \& Littlefield.

. 1998. Truth and progress. In his Philosophical papers, vol. 3. Cambridge: Cambridge University Press. . 1999. Philosophy and social hope. Harmondsworth, UK: Penguin.

Rorty, R., and J. Searle. 1999. Rorty v. Searle, at last: A debate. Logos 2 (3): 20-67. Rosenthal, S. 2000. Time, continuity, and indeterminacy: A pragmatic engagement with contemporary perspectives. Albany: State University of New York Press.

2003. The pragmatic reconstruction of realism: A pathway for the future. In Pragmatic naturalism and realism, edited by J. Shook. Amherst, NY: Prometheus.

Rouse, J. 1987. Knowledge and power: Toward a political philosophy of science. Ithaca, NY: Cornell University Press. 2003. Remedios and Fuller on normativity and science. Philosophy of the Social Sciences 33 (4): 464-71.

Ryder, J. 2003. Reconciling pragmatism and naturalism. In Pragmatic naturalism and realism, edited by J. Shook. Amherst, NY: Prometheus.

Ryle, G. 1949. The concept of mind. London: Hutchinson.

Searle, J. 1993. Rationality and realism, what is at stake? Daedalus 122 (4): 55-83. . 1995. The construction of social reality. New York: Free Press. 2001. Social ontology and the philosophy of society. In On the nature of social and institutional reality, edited by E. Lagerspetz, H. Ikäheimo, and J. Kotkavirta. Jyväskylä: SoPhi.

Sellars, W. 1956. Empiricism and the philosophy of mind. In The foundations of science and the concept of psychology and psychoanalysis, vol. 1 of Minnesota 
studies in the philosophy of science, edited by Herbert Feigl and Michael Scriven, 253-329. Minneapolis: University of Minnesota Press.

Sheehy, P. 2003. Social groups, explanations and ontological holism. Philosophical Papers 32 (2): 193-224.

Somers, M. 1998. "We're no angels": Realism, rational choice, and relationality in social science. American Journal of Sociology 104 (3): 722-84.

Tilly, C. 1984. Big structures, large processes, huge comparisons. New York: Russell Sage.

100 (6): 1594-610.

1995. To explain political processes. American Journal of Sociology 2001. Relational origins of inequality. Anthropological Theory 1

(3): $355-72$.

Valverde, M. 1998. Governing out of habit. Studies in Law, Politics, and Society 18: $217-42$.

Van Bouwel, J. 2004. Explanatory pluralism in economics: Against the mainstream? Philosophical Explorations 7 (3): 299-315. 26 Philosophy of the Social Sciences

Vandenberghe, F. 1999. "The real is relational": An epistemological analysis of Pierre Bourdieu's generative structuralism. Sociological Theory 17 (1): 32-67.

Wittgenstein, L. 1953. Philosophical investigations. Oxford: Blackwell. 